\title{
Implicaciones computacionales de los aeromanipuladores
}

\author{
Julio Mendoza-Mendoza ${ }^{1}$, Baltazar Jiménez-Ruiz ${ }^{2}$, \\ Víctor Javier González-Villela ${ }^{1}$, Mauricio Méndez-Martínez ${ }^{3}$, \\ Leonardo Fonseca-Ruiz ${ }^{3}$, Erick López Alarcón ${ }^{3}$ \\ 1 Centro de Ingeniería Avanzada, Facultad de Ingeniería, UNAM, \\ México \\ 2 Escuela Superior de Cómputo, Instituto Politécnico Nacional, \\ México \\ 3 Unidad Profesional Interdisciplinaria en Ingeniería y Tecnologías Avanzadas, \\ Instituto Politécnico Nacional, \\ México \\ aladegabriel@hotmail.com
}

\begin{abstract}
Resumen. Este documento presenta las necesidades computacionales implícitas en el diseño y operación de manipuladores de robots aéreos; para este propósito, se identifican y verifican diferentes consideraciones en el estado de la técnica actual y muy reciente. Se inicia con el cálculo requerido para las unidades UAS individuales, y continúa con la operación de estructura completa, que se divide en 4 modos de operación cíclicos: vuelo hacia el área de trabajo y transformacián a manipulador; despegue de aeronaves; control en modo manipulador y finalmente aterrizaje. A lo largo del texto, hay un ejemplo de aplicación, un brazo de robot volador desarrollado por los autores.
\end{abstract}

Palabras clave: manipuladores aerias robóticos, dise o, operación.

\section{Computational Implications of Aeromanipulators}

\begin{abstract}
This document presents the computational needs implicit in the design and operation of aerial-robot manipulators, for this purpose, different considerations are identified and verified in the current and very recent state of the art. It is started with the computation required for the individual UAS units, and continue with the full-structure operation which is divided into 4 cyclic modes of operation: flight towards the work area and transformation to manipulator; aircraft take off; control in manipulator mode and finally landing. Along the text, there is an example of application, a flying robot arm in development by the authors.
\end{abstract}

Keywords: aerial-robot manipulators, design, operation. 


\section{Introducción}

La tecnología de aeromanipuladores como estructuras voladoras de tipo brazo robot, tanto en configuraciones seriales como paralelas (Figura 1) data de 2012 $[1,2,3,4,5,6,7,8,9]$.

Básicamente se trata de estructuras aéreas donde los UAVs (acrónimo inglés para vehículo aéreo no tripulado) que las transportan, funcionan también como los elementos de manipulación robótica, para comprender las implicaciones computacionales relacionadas a estos mecanismos, es necesario subdividirlas en la siguientes áreas: 1 consideraciones computacionales generales de los UAVs; 2 consideraciones de transformación y vuelo hacia la zona de operación del sistema de manipulación aérea conjunta; 3 implicaciones computacionales de ascenso; 4 implicaciones computacionales de control en el modo de manipulación aérea; 5 implicaciones computacionales de descenso.

En nuestra aplicación particular, cada una de estas implicaciones es satisfecha vía hardware por el sistema embebido de vuelo Pixhawk y vía software mediante las librerías APM [10,11], sin embargo, este texto resultará de utilidad para quien desee hacer su propio sistema con microprocesadores, FPGAS, DSPs, microcontroladores o tarjetas de desarrollo de altas prestaciones. Para el correspondiente modelado y condiciones de operación ver [9].

\section{Implicaciones generales}

Las implicaciones computacionales generales de los vehículo aéreo no tripulado, se recogen en $[12,13,14,15]$.

Cada uno de los elementos descritos posee distintas frecuencias de operación por lo cual es necesario un sistema de administración RTOS (real time operating system) que dictamine la adecuada lectura, procesamiento, almacenamiento, jerarquía y ejecución, estos consisten en:

Lectura de sensores de orientación, posición planar y altura: La manera estándar es utilizar IMUs, magnetómetros, barómetros, acelerómetros y GPS o cámaras, todos operando en distintos protocolos de comunicación y frecuencias de muestreo, en algunos casos por construcción del sensor y en otros por prioridad de medición.

Escritura a propelas y mecanismos de ejecución mediante servomotores: Lo primero es el uso directo con motores generalmente brushless (sin escobillas) y lo segundo consiste en utilizar motores auxiliares, para tareas secundarias al vuelo, por ejemplo estabilizadores de cámara, alerones y mecanismos de thrust vectoring (vectorización del empuje principal) $[16,17,18]$. Esto es importante porque la escritura a los primeros debe ser en frecuencias mucho mayores a los segundos, basicamente porque de los primeros depende que no caiga la aeronave y están sujetos a altas vibraciones, estos por lo general deben operar a PWMs (acrónimo inglés para modulación por ancho de pulsos) de 490 $\mathrm{Hz}$ mientras que los secundarios a $50 \mathrm{~Hz}$.

Control retroalimentado de orientación, posición planar y altura: De manera general, se subdivide la tarea de movimiento de un UAV en estos tres 
modos de ejecución, el prioritario es mantener una altura de operación también conocido como hovering; el de rango de prioridad intermedia es controlar la orientación o actitud de la aeronave y finalmente su posición planar, también conocida como steering $[19,20,21,15,22]$.

Procesamiento de imágenes: Esta tarea se vuelve necesaria en aquellas condiciones donde no funcionan los sistemas de posicionamiento global satelital (por ejemplo en interiores, o zonas rocosas o boscosas).

Comunicación interna de datos de entrada y salida: Esta por lo general se realiza en un bus de topología serial, como se indica consiste en la forma en la cual se comparten y procesan los datos de los sensores hacia el procesador principal y a la vez el modo en el que este decide su escritura a los diversos sistemas de actuadores.

Almacenamiento de datos de vuelo y telemetría: Es el modo en el que el sistema almacena los datos para su uso e interacción y el modo de comunicación inalámbrica entre el mando de control autónomo principal.

Lectura de mandos de control remoto: Consiste en la lectura de unidades de teleoperación, las cuales establecen el vinculo entre la unidad en vuelo y la base manual en tierra, como puede verse, junto con la telemetría existen dos distintos módulos de mando inalámbrico, el reto aquí es garantizar que operen con precisión en frecuencias distintas y en múltiples canales independientes.

Lectura de unidades analógicas y digitales tipo GPIO: Consiste en la habilitación, interacción, lectura y escritura de los puertos analógicos o bien aquellos digitales de propósito general (general purpose input/output).

Filtrado de señales: La adición de ruido a los UAVs esta implícita en la vibración de los motores y su interacción eléctrica, de esta forma, es necesario un algoritmo de filtrado de señales, el cual generalmente es mediante técnicas.

Fusión sensorial: Cuando el medio cambia de manera constante, es necesario utilizar combinación de sensores de geolocalización, un ejemplo es entre tareas donde el dron pase de un ambiente externo a un interno y se deba también intercambiar entre la lectura de un sistema de visión artificial y un sensor GPS.

Planificación de tareas: Es necesario obtener datos de velocidad y aceleración para disponer de movimientos suaves, mas aun, hay autores que indican y demuestran que es necesario incluir snap (segunda derivada de aceleración) [23], como los sensores se limitan a lectura de velocidades posiciones y aceleraciones y en la mayoría de los casos es parcialmente, es necesario incluir estimadores que implican observadores, estimación neuronal, estimación difusa y a su vez algoritmos de planificación de trayectorias entre puntos, lo cual, también vuelve necesario incluir algoritmos de optimización de productos matriciales.

Prioridad de tareas en tiempo real: Dadas las diferentes frecuencias de operación de sensores, actuadores, sistemas y sub-lazos de control, es necesario priorizar las tareas de ejecución, las de mayor prioridad suelen ser aquellas de escritura a motores y lectura de altura; las intermedias las de lectura planar y orientacional y finalmente en menor rango las de comunicación serial, lectura de mandos remotos y almacenamiento de datos de vuelo, ahora bien dentro de las prioritarias relacionadas a escritura de motores, las jerarquía es altitud, actitud, 
movimiento de plano y trayectoria espacial, el Pixhawk dispone para ello de un sistema RTOS para asignar prioridades y tiempos, sin embargo en caso de diseñar su propia controladora de vuelo, es necesario establecer un sistema de tiempo real para ejecución de eventos.

\section{Máquina de estados del vuelo de un aeromanipulador, caso particular aerobrazo}

Antes de proseguir con las áreas de implicaciones computacionales es adecuado indicar la máquina de estados relacionada a la operación del aerobrazo [9], para ello se subdivide la operación conjunta en dos etapas: 1 traslado y transformación: es decir la operación conjunta del sistema de UAVs para llegar a la zona de operación y una vez ahí transformarse en manipulador aéreo; 2 manipulación aérea: es decir anclar su posición en la zona de operación deseada y funcionar como brazo robótico flotante:

A la izquierda de la Figura 3 se ilustra la ejecución de la máquina de estados, el lazo mas externo es la transformación entre aeronave y brazo volador el cual corresponde a la activación o desactivación de un sistema tipo clutch, cuando el clutch esta activo, los UAVS tienen un comportamiento de estructura rígida y solo contribuyen al traslado de la misma, una vez que esta desactivado el clutch, lo cual esta en función de la zona de operación donde se desea el modo de manipulador aéreo, se inicia el modo de manipulación aérea.

El modo de manipulación aérea, es decir el lazo interno de la máquina de estados, esta basado en el hecho de que se poseen dos controles remotos y puede compartirse su mando auxiliar entre ambos drones mediante protocolos alambricos o inalambricos Figura 2, de esta manera cada botón auxiliar tiene dos estados 1 o encendido y 0 o apagado, al ser dos mandos existen entonces 4 combinaciones (00), (01), (11 )y (10), que respectivamente indicarían la secuencia de apagado, ascenso, control y descenso respectivamente, la maquina de estados deseable para nuestros propósitos es la siguiente: estando en el modo apagado (00) solo se admite trasladarse al estado de ascenso (01), cualquier otra combinación mantiene al sistema en el modo de apagado. Una vez ascendiendo (01) solo se admite el paso al modo de control de altura (11) y ángulos de hombro y codo o bien al modo de descenso (10). En el modo de control (11) solo es admisible pasar al modo de descenso (10). Del modo de descenso (10) solo es admisible cambiar al modo de apagado (00).

\section{Implicaciones computacionales de vuelo y transformación hacia la zona de trabajo, modo aeronave}

Coordinación y programación paralela de vuelo físicamente restringido de múltiples UAVS: La cual puede ser centralizada si se usa un solo procesador y se ve al sistema no como un conjunto de UAVS sino como un 
conjunto de hélices o bien descentralizada (multiprocesador) si cada UAV usa su respectivo procesador y se ve al sistema como un grupo coordinado de UAVs autónomos, la tarjeta Pixhawk es capaz de realizar ambos modos de operación con UAVs de hasta 4 rotores para una configuración de aerobrazo de 8 hélices (es decir 2 cuadrotores o bien 4 coaxiales de dos hélices u ocho monocopteros). El problema de la operación centralizada es la sobrecarga de tareas de actuación y control para un solo procesador.

Estimación de perturbaciones en vivo: Aunque todo el vuelo posee perturbaciones, la parte critica es aquella de larga duración, es decir en modo aeronave, donde se vuelve necesario estimar y contrarrestar los efectos del viento, la presión y temperatura, esto es computacionalmente complicado a cielo abierto pues el aire representa un flujo laminar y varia con modelos diferenciales parciales que inciden sobre variaciones de una viga (la aeronave se considera como una viga múltiplemente apoyada sobre sus hélices).

Lectura simultanea de múltiples puntos para determinar parámetros de inercia y masa en un punto virtual: Dicho punto virtual es el centro de gravedad o de masas el cual es virtual porque puede o no estar físicamente en el cuerpo del sistema, pero es de suma importancia para el control del mismo, como este punto dependen de la configuración de cada UAV, un buen algoritmo para determinarlo esta en [24] el cual implica calcular las variaciones inerciales de cada UAV y/o punto intermedio de unión y transferirlas a una central de mando para calcular así el punto inercial equivalente, esto computacionalmente implica el envió de datos sensoriales simultáneos a una central de procesamiento donde se procesan y a su vez se devuelve el punto resultante a cada UAV para que tengan noción de la operación conjunta, ello implica envió por protocolos alambricos o inalambricos, generalmente seriales y a su vez métodos para asegurar la adecuada transferencia de la información (el mas básico es el cheksum).

Interacción de transformación: En este punto, es necesario un método para transformar la estructura aérea desde un modo de vuelo estándar y coordinado (por ejemplo un helicóptero o un ala volante) a uno de vuelo independiente pero igualmente coordinado (es decir un brazo volador), la implicación computacional es la conmutación de frecuencias de operación de las helices las cuales deben apagarse y encenderse en una secuencia precisa mientras el sistema vuela (helibot patente MX/a/2016/014595) o bien [6,9], vase Figura 1.

\section{Implicaciones computacionales de ascenso modo compartido}

El ascenso es la parte mas simple pero peligrosa de la operación de un sistema aéreo, esto es porque primero se debe garantizar un despegue el cual no ocurre de manera inmediata a la activación de las hélices, es decir existe un bias en el cual las hélices giran pero el sistema no consigue elevación, mas aun cada hélice posee su propio bias de despegue en dependencia del adecuado centrado de todo el sistema o bien el balanceo de cada propela, la implicación computacional en esta parte consiste en monitorear un valor optimo para la elevación conjunta sin 

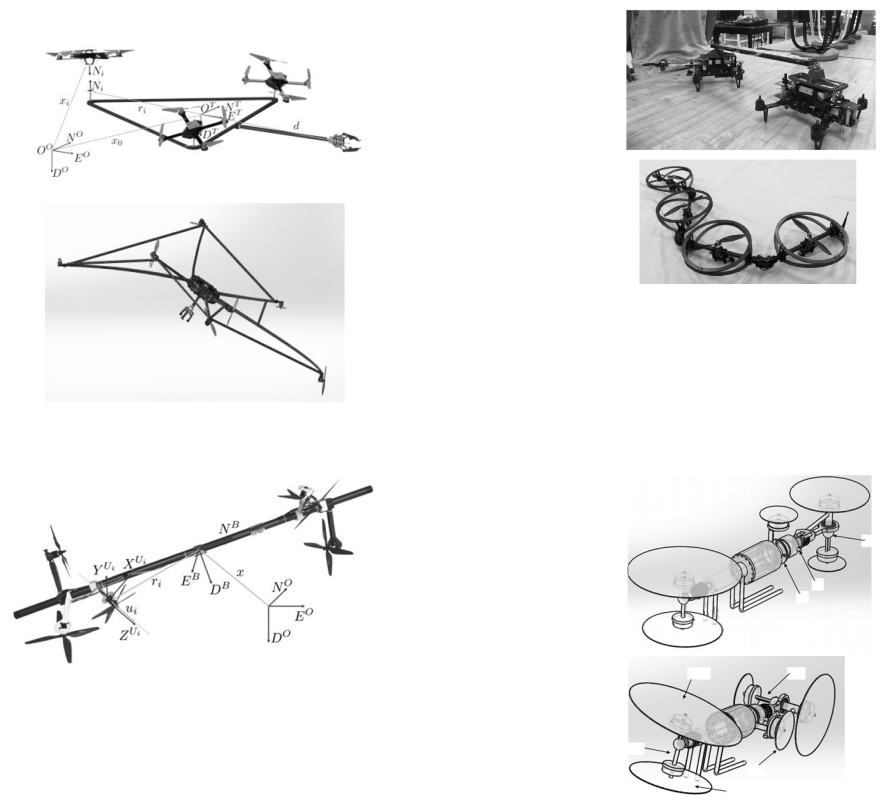

Fig. 1. Aeromanipuladores: De arriba a abajo y de izquierda a derecha: Hai-Nguyen 2015; Julio Mendoza 2015; Alexandros Nikou 2015; Moju Zhao 2016; Sangyul Park 2016; Mendoza MX/a/2016/014595 MX/a/2015/015160.
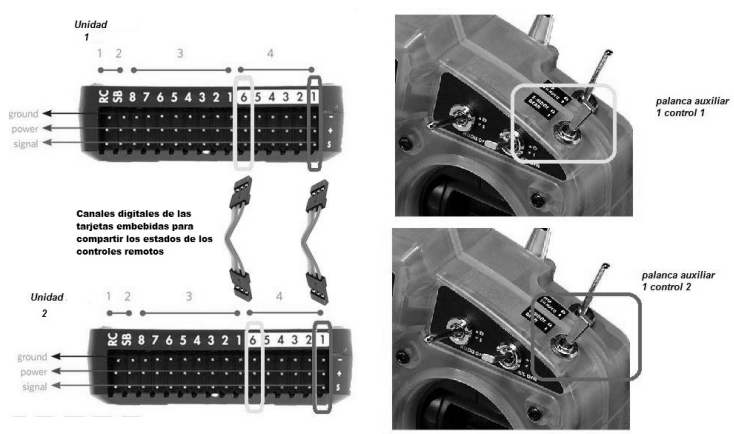

Fig. 2. Comunicación alambrica entre UAVs hombro y codo. 


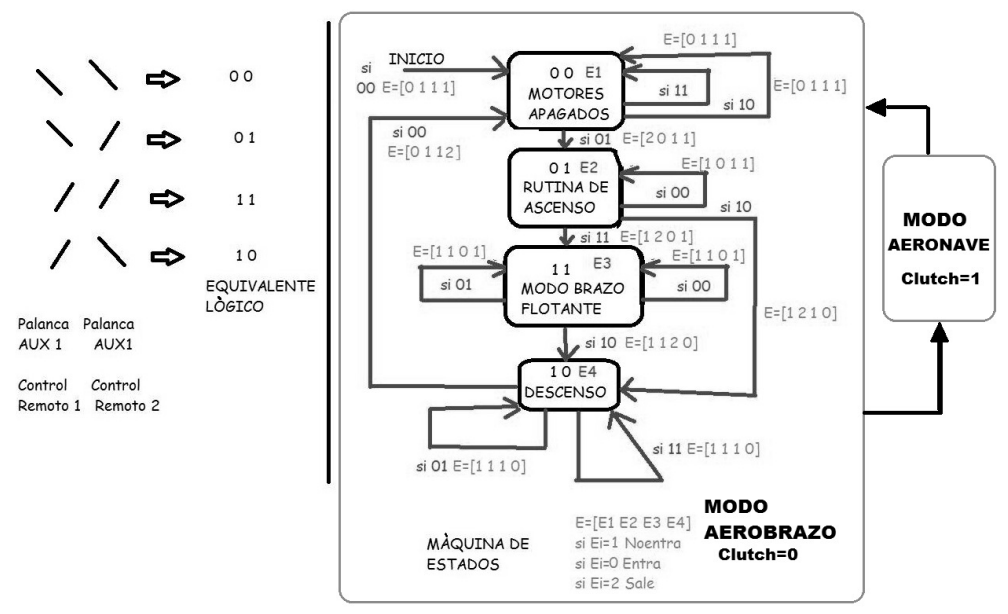

Fig. 3. Máquina de estados del aerobrazo.

llegar al nivel de apagado y también dictaminar un valor en el cual se mude al estado de control.

\section{Implicaciones computacionales de control modo aeromanipulador}

Restricciones de giro en los motores: Una vez activado el modo de control, surgen dos importantes implicaciones computacionales, la primera guarda relación al cambio de giro de los motores, es decir mientras el control puede alcanzar cotas positivas y negativas, algunas aeronaves como los cuadricopteros exigen que sus motores no cambien de sentido de giro (para evitar efectos de autorotación), por lo tanto se debe recurrir al problema de optimización descrito en $[8,5]$ :

El cual simplemente expresa eso, el control aplicado a los motores debe mantenerse positivo pese a la variación del error y el control de vuelo.

Ahora bien en caso de disponer variación de sentido de giro, esta no puede realizarse arbitrariamente y sigue consignas de absorción de resultantes, una manera es agregar un motor adicional que contrarreste vectorialmente todas las variaciones negativas de la señal de control de los demás motores [3].

Jerarquía y calculo en linea del control: La segunda consiste en recalcular el propio control, el cual como se dijo es de tres tipos, elevación, actitud y plano y saber en que situaciones resulta adecuado dotar de mas importancia a uno respecto a otro, como se dijo el control mas importante es el de altura, pero paradójicamente en la elevación del dron, dada la desigualdad en el bias de empuje de cada motor, el mas importante es el de actitud, otro ejemplo es en las tareas acrobáticas y agresivas $[25,23]$ en este caso se debe discernir y diseñar si 
la tarea tiene prioridad por la conservación de ángulo o por la de posición planar [26].

Optimización de sobreactuación: Otra implicación computacional de control es la de sobreactuación (mas motores que grados de libertad): en este caso la matriz que transfiere el control cartesiano 6D (3 dimensiones de traslación y 3 de rotación), debe transformarse a control de velocidades en cada propela, si por ejemplo tenemos un sistema de 2 cuadricopteros, son 8 propelas por lo cual las matrices de transformación exigen métodos de optimización como los basados en pseudoinversas y además respetar las restricciones descritas en los párrafos precedentes.

\section{Implicaciones computacionales de descenso modo compartido}

El descenso es otra operación simple pero delicada, consiste en apagar paulatinamente los motores pero cuidando que estos se mantenga en su modo de sustentación (es decir que posean suficiente fuerza para garantizar que el sistema no se desplome), como fue dicho, los motores no tienen un bias homogéneo por lo cual el control de orientación cobra mayor prioridad en este modo de operación, tal y como es exagerado en el cine y la tv, el proceso implica escalones de encendido y apagado, cuando se nota un desplome severo, se aplica un impulso de elevación y así hasta tocar suelo, la complejidad computacional consiste en mantener operativos los sensores y actuadores en tiempos precisos que garanticen un descenso suave y además no destructivo.

\section{Conclusiones}

En este documento se muestran las implicaciones computacionales mas frecuentes encontradas en el diseño del aerobrazo y también encontradas en proyectos semejantes $[3,5,6]$, estas se subdividen en distintas tareas y se muestran al lector como un conjunto de módulos a considerar y resolver si se pretende realizar manipulación aérea coordinada.

Asimismo se da énfasis en la necesidad de poseer sistemas RTOS desde nivel moderado hasta estricto en función de la tarea a ejecutarse, de esta forma se sugiere utilizar sistemas aéreos de navegación embebida como el Pixhawk, Erlebrain o NavIO, microcontroladores dedicados o bien FPGAs o microprocesadores y decantar opciones de tarjetas de desarrollo básicas (Arduino, Raspberry), de hecho este fue el motivo de la evolución del APM al Pixhawk, el primero basado en un Arduino y el segundo basado en un microprocesador Cortex M4F.

Agradecimientos. Los autores agradecen a la Secretaría de Investigación y Posgrado del Instituto Politécnico Nacional, y sus correspondientes proyectos SIP y BEIFI, asimismo a la DGAPA-UNAM y a COFAA-IPN. 
Implicaciones computacionales de los aeromanipuladores

\section{Referencias}

1. Tognon, M., Franchi, A.: Control of motion and internal stresses for a chain of two underactuated aerial robots. In: Control Conference (ECC), 2015 European, IEEE (2015) 1620-1625

2. Naldi, R., Forte, F., Marconi, L.: A class of modular aerial robots. In: Decision and Control and European Control Conference (CDC-ECC), 2011 50th IEEE Conference on, IEEE (2011) 3584-3589

3. Nikou, A., Gavridis, G.C., Kyriakopoulos, K.J.: Mechanical design, modelling and control of a novel aerial manipulator. In: Robotics and Automation (ICRA), 2015 IEEE International Conference on, IEEE (2015) 4698-4703

4. Mellinger, D., Shomin, M., Michael, N., Kumar, V.: Cooperative grasping and transport using multiple quadrotors. In: Distributed autonomous robotic systems. Springer (2013) 545-558

5. Park, S., Her, J., Kim, J., Lee, D.: Design, modeling and control of omni-directional aerial robot. In: Intelligent Robots and Systems (IROS), 2016 IEEE/RSJ International Conference on, IEEE (2016) 1570-1575

6. Zhao, M., Kawasaki, K., Chen, X., Kakiuchi, Y., Okada, K., Inaba, M.: Transformable multirotor with two-dimensional multilinks: Modeling, control, and whole-body aerial manipulation. In: International Symposium on Experimental Robotics, Springer (2016) 515-524

7. Nguyen, H.N., Park, S., Lee, D.: Aerial tool operation system using quadrotors as rotating thrust generators. In: Intelligent Robots and Systems (IROS), 2015 IEEE/RSJ International Conference on, IEEE (2015) 1285-1291

8. Choi, F.: Modeling and control of asymmetrically coupled quadrotor uavs. Master's thesis, Seoul National University (2015)

9. Mendoza-Mendoza, J., Sepulveda-Cervantes, G., Aguilar-Ibanez, C., Mendez, M., Reyes-Larios, M., Matabuena, P., Gonzalez-Avila, J.: Air-arm: A new kind of flying manipulator. In: Research, Education and Development of Unmanned Aerial Systems (RED-UAS), 2015 Workshop on, IEEE (2015) 278-287

10. authors, S.: Pixhawk webpage. https://pixhawk.org/ (2010) Acces 18-08-2017.

11. authors, S.: Ardupilot libraries webpage. http://ardupilot.org/dev/docs/apmcopterprogramming-libraries.html (2010) Acces 18-08-2017.

12. Meier, L., Tanskanen, P., Fraundorfer, F., Pollefeys, M.: Pixhawk: A system for autonomous flight using onboard computer vision. In: Robotics and automation (ICRA), 2011 IEEE international conference on, IEEE (2011) 2992-2997

13. Fresk, E., Nikolakopoulos, G., Gustafsson, T.: A generalized reduced-complexity inertial navigation system for unmanned aerial vehicles. IEEE Transactions on Control Systems Technology 25(1) (2017) 192-207

14. Santana, L.V., Brandao, A.S., Sarcinelli-Filho, M.: Outdoor waypoint navigation with the ar. drone quadrotor. In: Unmanned Aircraft Systems (ICUAS), 2015 International Conference on, IEEE (2015) 303-311

15. Quan, Q.: INTRODUCTION TO MULTICOPTER DESIGN AND CONTROL. Springer (2017)

16. Páscoa, J., Dumas, A., Trancossi, M., Stewart, P., Vucinic, D.: A review of thrustvectoring in support of a v/stol non-moving mechanical propulsion system. Open Engineering 3(3) (2013) 374-388

17. Rajappa, S., Ryll, M., Bülthoff, H.H., Franchi, A.: Modeling, control and design optimization for a fully-actuated hexarotor aerial vehicle with tilted propellers. In: Robotics and Automation (ICRA), 2015 IEEE International Conference on, IEEE (2015) 4006-4013 
18. Ryll, M., Bülthoff, H.H., Giordano, P.R.: Modeling and control of a quadrotor uav with tilting propellers. In: Robotics and Automation (ICRA), 2012 IEEE International Conference on, IEEE (2012) 4606-4613

19. Carrillo, L.R.G., López, A.E.D., Lozano, R., Pégard, C.: Quad rotorcraft control: vision-based hovering and navigation. Springer Science \& Business Media (2012)

20. Valavanis, K.P., Vachtsevanos, G.J.: Handbook of unmanned aerial vehicles. Springer Publishing Company, Incorporated (2014)

21. Schafroth, D., Bermes, C., Bouabdallah, S., Siegwart, R.: Micro helicopter steering: Review and design for the mufly project. In: Mechtronic and Embedded Systems and Applications, 2008. MESA 2008. IEEE/ASME International Conference on, IEEE (2008) 216-221

22. Torenbeek, E., Wittenberg, H.: Flight physics: essentials of aeronautical disciplines and technology, with historical notes. Springer Science \& Business Media (2009)

23. Mellinger, D., Kumar, V.: Minimum snap trajectory generation and control for quadrotors. In: Robotics and Automation (ICRA), 2011 IEEE International Conference on, IEEE (2011) 2520-2525

24. Al-Rawashdeh, Y.M., Elshafei, M., Al-Malki, M.F.: In-flight estimation of center of gravity position using all-accelerometers. Sensors 14(9) (2014) 17567-17585

25. Lee, T., Leoky, M., McClamroch, N.H.: Geometric tracking control of a quadrotor uav on se (3). In: Decision and Control (CDC), 2010 49th IEEE Conference on, IEEE (2010) 5420-5425

26. Mellinger, D., Michael, N., Kumar, V.: Trajectory generation and control for precise aggressive maneuvers with quadrotors. The International Journal of Robotics Research 31(5) (2012) 664-674 